\title{
Solving the Stefan problem for a solid phase growth on plane plate and spherical surfaces and testing of theoretical equations
}

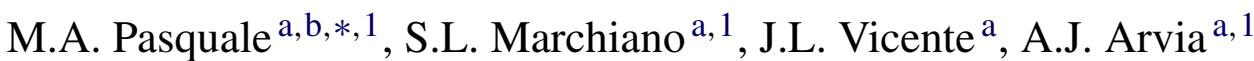 \\ ${ }^{a}$ Instituto de Investigaciones Físicoquimicas Teóricas y Aplicadas (INIFTA), Universidad Nacional de La Plata-Consejo Nacional de \\ Investigaciones Científicas y Técnicas, Sucursal 4, Casilla de Correo 16, (1900) La Plata, Argentina \\ ${ }^{\mathrm{b}}$ Facultad de Ciencias Fisicomatemáticas e Ingeniería, Pontificia Universidad Católica Argentina, Buenos Aires, Argentina
}

Received 3 September 2005; received in revised form 29 October 2005; accepted 5 November 2005

Available online 15 December 2005

\begin{abstract}
Solutions of the Stefan problem in the 2D space considering a moving boundary of a solid deposit growing under mass transfer control on either plane plate or spherical solid substrates are reported. In the former case, the displacement of the growth front at the plane plate occurs perpendicularly to the substrate, whereas for the latter it shifts radially. For both substrates, in the absence of convection and surface roughness effects, the phase growth kinetics is determined by diffusion and advection, the latter being due to the linear displacement of the growth front with time. For both geometric arrangements the theory predicts two limiting kinetic situations, namely a diffusion control when the time and/or the radius of the substrate approach zero, and an advection control for the reverse conditions. For the spherical substrate, when its radius tends to infinity, the kinetics of the process approaches that found at the plane plate substrate. Theoretical potentiostatic current density transients are tested utilising growth pattern data for the formation of 2D silver dense branching electrodeposits on a plane plate cathode in a quasi-2D cell, and silver electrodeposits on spherical cathodes employing a high viscosity plating solutions.
\end{abstract}

(C) 2005 Elsevier Ltd. All rights reserved.

Keywords: Stefan problem; Diffusion-advection; Plane plate and spherical electrode; Silver electrodeposition

\section{Introduction}

Most rate equations that have been derived solving the convective-diffusion differential equations for the growth of a solid phase at a solid substrate have considered that the interface remains at rest [1]. This implies a limitation to the extension of those rate equations to processes in which the solid phase growth front moves inward (forward) into the bulk of the reactant supplier environment [2]. In this case, the solution of the corresponding differential equations depends on the moving boundary conditions [3].

Since about a century ago, solutions of moving boundary problems have been proposed for heat transfer in iceberg displacement [4-6], although their extension to mass transfer processes, such as those involved in solid phase growth, has received much less attention. These mass transfer problems are generi-

\footnotetext{
* Corresponding author. Tel.: +54 221 4257439; fax: +54 2214254642.

E-mail address: miguelp@inifta.unlp.edu.ar (M.A. Pasquale).

1 ISE member.
}

cally denoted as Stefan problems, irrespective of the driving force. They are often found in a number of processes occurring in different areas of natural sciences and technologies.

In principle, both Fick's and Fourier's equations can be solved in each phase for different situations. (i) For a fixed boundary the transfer equations are solved for the immobile interface assuming constant spatial domains for each phase. (ii) For a moving boundary the spatial domain of each phase changes as the interface front moves according to a certain law that is known a priori. (iii) For an implicit free boundary the spatial domain changes and the moving boundary equations are unknown. They usually depend on the physics of each problem and have to be found in order to solve them [7].

This paper compares two solutions of the Stefan problem in the 2D space considering the moving boundary of solid phase growth [case (ii)] on either a plane plate or a spherical solid under mass transfer control. In the former case, the displacement of the growth front occurs perpendicularly to the substrate surface, whereas for the latter it shifts radially. For this case, when the radius tends to infinity, the limiting solution of the mass transfer differential equation approaches the solution for the plane plate 
substrate. Neither changes in the macroscopic roughness of the moving boundary nor convective effects from density gradients are considered in solving the transfer equations.

On the other hand, theoretical potentiostatic current density transients are tested with experimental data related to the electrochemical formation of silver dense branching patterns under mass transfer control on plane plate and spherical cathodes. For this purpose, to make the contribution of free convection negligible, in the former case a quasi-2D cell and conventional plating solutions are utilised, whereas for the latter high viscosity plating solutions are employed. After correction for the roughness of the electrodeposits, the agreement of theoretical and experimental data in the time range where the contribution of advection is dominant is fairly good.

\section{The solution of Fick's equation with the Stefan boundary condition}

\subsection{Plane plate substrate}

Let us consider the deposition of species $i$ on a plane plate substrate of infinite dimensions by a diffusion process from a fluid phase (plating solution) that occurs normally ( $y$-direction) to the plane plate. This process is expressed by Fick's equation in Cartesian coordinates

$D_{i} \frac{\partial^{2} c_{i}}{\partial y^{2}}=\frac{\partial c_{i}}{\partial t}$

$D_{i}$ and $c_{i}$ being the diffusion coefficient and the concentration of species $i$ (reactant) in the fluid phase and $t$ is the deposition time. The advance of the growth front occurs in the $y$-direction. Eq. (1) is solved with the following boundary conditions:

$c_{i}(y, 0)=c_{i}^{0} ; \quad y \geq 0 ; \quad s(t)=s(0)$

$c_{i}[s(t), t]=0 ; \quad t>0$

$s(t)=\beta \cdot t$

$c_{i}(y, t)=c_{i}^{0} ; \quad y \rightarrow \infty$

Condition (2a) corresponds to the initially uniform reactant concentration in the plating solution, and $s(t)$ is the front coordinate at time $t$. The surface area of the growth front is equal to that of the inert substrate upon which the growth of the solid phase commences $(t=0) ; \beta$ denotes the advance velocity of the moving boundary. Condition ( $2 \mathrm{~b})$ indicates that the concentration of $i$ just at the growing front is null for $t>0$, as expected from mass transfer-controlled kinetics. Condition (2c), usually called the Stefan condition, indicates the instantaneous location of the growing front.

Solving Eq. (1) with boundary conditions ((2a)-(2d)), the explicit expression for $c_{i}(y, t)$ results in

$$
\begin{aligned}
c_{i}= & c_{i}^{0}-\frac{c_{i}^{0}}{2}\left\{\exp \left[\left(\frac{\beta}{D_{i}}\right) \cdot(\beta t-y)\right]\right. \\
& \left.\times\left[1-\operatorname{erf}\left(\frac{y-2 \beta t}{2 \sqrt{D_{i} t}}\right)\right]+1-\operatorname{erf}\left[\frac{y}{2 \sqrt{D_{i} t}}\right]\right\}
\end{aligned}
$$

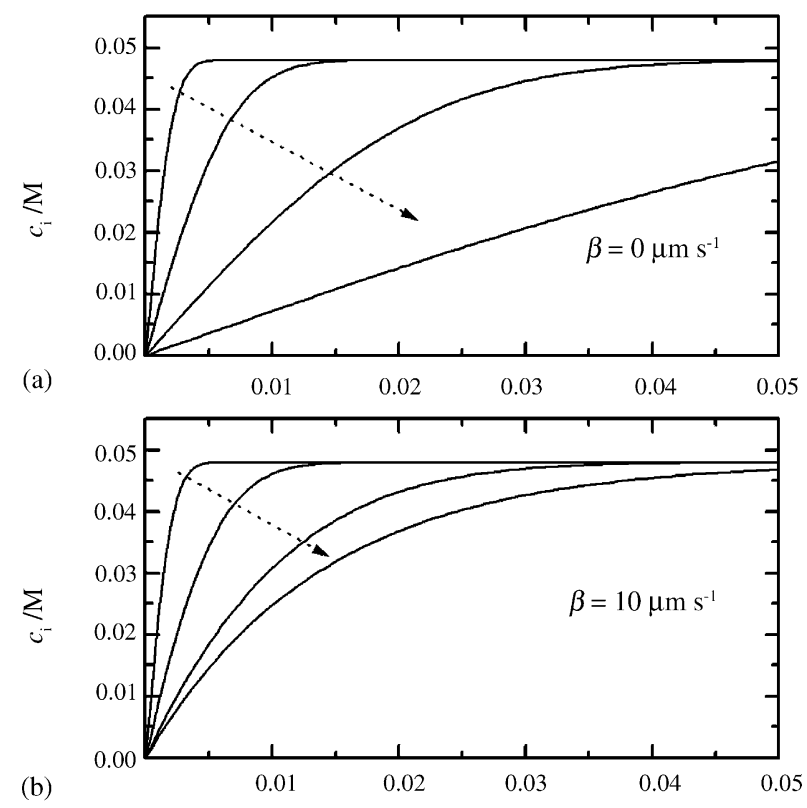

(b)

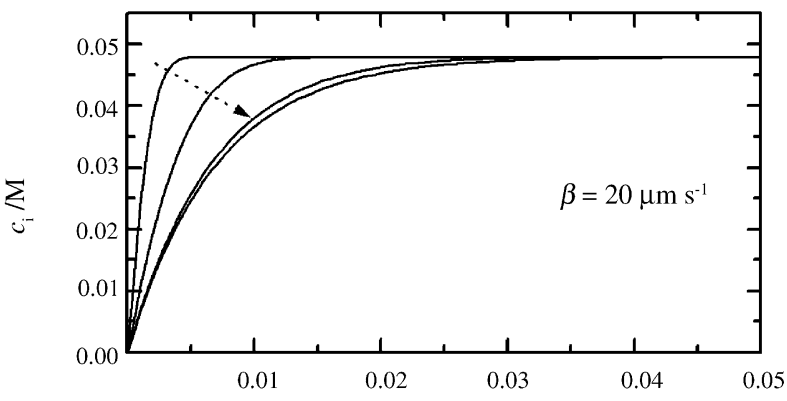

(c)

$(\mathrm{y}-\beta \mathrm{t}) / \mathrm{cm}$

Fig. 1. (a-c) Concentration profiles calculated for the plate plane with Eq. (3) plotted as $c_{i}$ vs. $y-\beta t$ at different values of $\beta$ for $t=0.1,1,10$ and $100 \mathrm{~s}$. The value of $t$ increases as indicated by the arrows. The following values were used in the calculations: $z_{i}=1 ; D_{i}=1.39 \times 10^{-5} \mathrm{~cm}^{2} \mathrm{~s}^{-1} ; c_{i}^{0}=4.8 \times 10^{-5} \mathrm{~mol} \mathrm{~cm}^{-3}$. These figures were taken from the electrodeposition of silver from aqueous solution [9].

Eq. (3) fulfills boundary conditions (2a) and (2b). Plots of $c_{i}$ versus $y-\beta t$ for various values of $\beta$ and different values of $t$ are shown in Fig. 1. At constant $y-\beta t$ the gradient of $c_{i}$ perpendicular to the substrate increases with $\beta$. This effect is more remarkable at longer $t$.

Let us apply Eq. (3) to evaluate the potentiostatic current density transients, which represent the instantaneous rate of metal electrodeposition from the plating solution on the plane plate. The rate of this process under diffusion control is given in terms of the cathodic current density $j_{\mathrm{c}}(t)$

$j_{\mathrm{c}}(t)=z_{i} F D_{i}\left[\frac{\partial c_{i}(y, t)}{\partial y}\right]_{y=\beta t}$

$z_{i}, c_{i}$ and $F$ being the electric charge per reactant species, the reactant concentration in the plating solution and the Faraday constant, respectively. Then, considering the differential expres- 


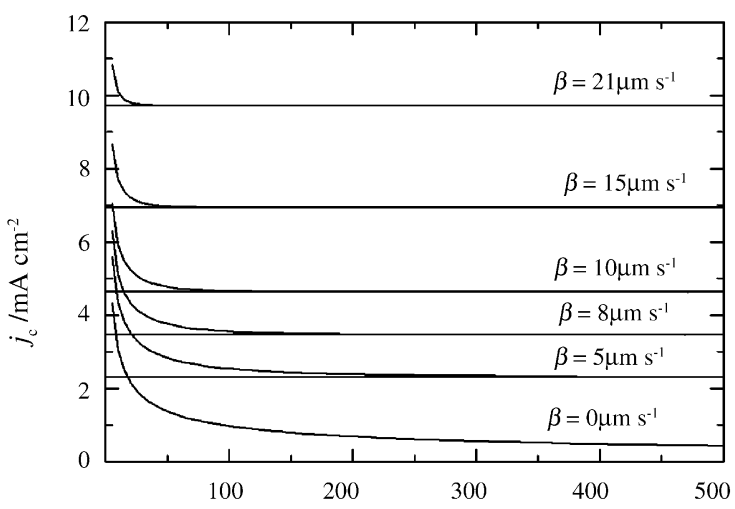

(a)

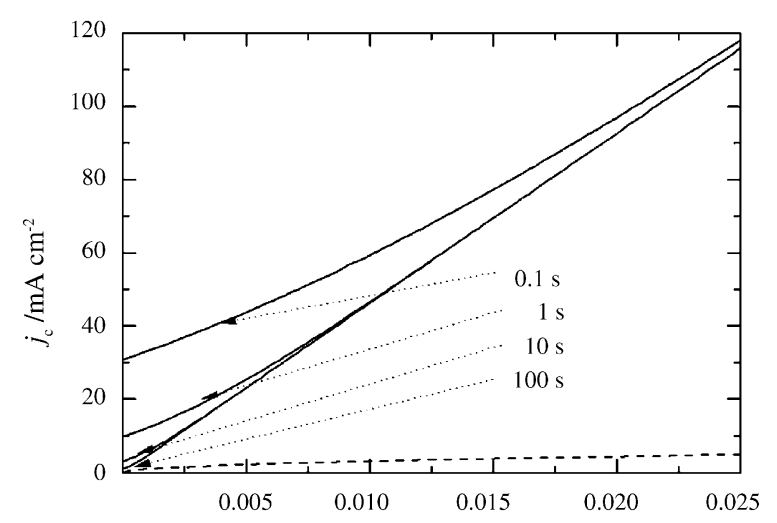

(b)

$$
\beta / \mathrm{cm} \mathrm{s}^{-1}
$$

Fig. 2. (a) Plots of $j_{\mathrm{c}}$ vs. $t$ calculated from Eq. (5) at different values of $\beta$, as indicated. The height of the advection plateau increases with $\beta$. The same values of $z_{i}, D_{i}$ and $c_{i}^{0}$ indicated in Fig. 1 were used. (b) Plots of $j_{\mathrm{c}}$ vs. $\beta$ calculated from Eq. (5) for different $t$, as indicated. Values of $z_{i}, D_{i}$ and $c_{i}^{0}$ are the same as those indicated in Fig. 1. The dashed trace (at the bottom) corresponds to the $j_{\mathrm{c}}$ vs. $\beta^{1 / 2}$ plot resulting for laminar flow forced convection [1].

sion of Eq. (3), for $j_{\mathrm{c}}(t)$ one obtains

$$
\begin{aligned}
j_{\mathrm{c}}(t)= & z_{i} F c_{i}^{0}\left\{\left(\frac{D_{i}}{\pi t}\right)^{1 / 2} \exp \left[\left(-\frac{\beta^{2} t}{4 D_{i}}\right)\right]\right. \\
& \left.+\frac{\beta}{2}\left[\operatorname{erf}\left[\frac{\beta}{2}\left(\frac{t}{D_{i}}\right)^{1 / 2}\right]+1\right]\right\}
\end{aligned}
$$

The first RHS term in Eq. (5) is the product of the diffusion term for a fixed plane plate and an exponential factor. The latter decreases the value of $j_{\mathrm{c}}(t)$ faster than that predicted by the solution of Fick's equation for a fixed boundary [1]. The contribution of the first term becomes more remarkable for $t \rightarrow 0$. The greater the value of $\beta$, the faster the decay of $j_{\mathrm{c}}(t)$ (Fig. 2a). The second $\beta$-dependent RHS term increases the value of $j_{\mathrm{c}}(t)$ even for $t=0$. The limit of Eq. (5) for $t \rightarrow 0$ is

$\left[j_{\mathrm{c}}(t)\right]_{t \ll 1}=z_{i} F c_{i}^{0}\left[\sqrt{\frac{D_{i}}{\pi t}}+\frac{\beta}{2}\right]=z_{i} F c_{i}^{0} \sqrt{\frac{D_{i}}{\pi t}}+\frac{\left(j_{\mathrm{c}}\right)_{\mathrm{adv}}}{2}$

The second term for $t \rightarrow \infty$ leads to a steady regime that corresponds to advection control [8,10]. The limit of Eq. (5) for $t \rightarrow \infty$ is

$\lim \left[j_{\mathrm{c}}(t)\right]_{t \rightarrow \infty}=z_{i} F c_{i}^{0} \beta=\left(j_{\mathrm{c}}\right)_{\mathrm{adv}}$

Eq. (7) corresponds to the advection limit to the mass transport process. The greater the value of $\beta$, the faster the advection regime is reached (Fig. 2b).

The linear dependence of $j_{\mathrm{c}}$ on $\beta$ from Eq. (7) contrasts with the $\langle\beta\rangle^{1 / 2}$ dependence that would result, in general, from laminar flow forced convection $[1,11]$. Therefore, the different hydrodynamic regimes that might be involved in the process can be clearly distinguished (Fig. 2b).

On the other hand, Eq. (5) predicts a kinetic transition in the electrodeposition process from a non-steady planar diffusion Stefan process to a steady advection regime.

To further explore the behavior of Eq. (5), let us define the dimensionless current ratio $\rho(\tau)$

$\rho(\tau)=\frac{j_{\mathrm{c}}(\tau)}{\left(j_{\mathrm{c}}\right)_{\mathrm{adv}}}$

and the dimensionless variable $(\tau)$

$\tau=\frac{\beta^{2} t}{4 D_{i}}$

Then, by replacing Eqs. (5) and (7) into Eq. (8), and further considering the dimensionless variable $\tau$ from Eq. (9), the following dimensionless equation is obtained:

$\rho(\tau)=\frac{1}{2}\left[\frac{1}{\sqrt{(\pi \tau)}} \exp (-\tau)+\operatorname{erf}(\sqrt{\tau})+1\right]$

Obviously, due to the contribution of advection in Eq. (10), for $\tau \rightarrow \infty$ it results in $\rho(\tau) \rightarrow 1$.

\subsection{Spherical substrate}

Let us consider the same type of diffusion process as the above that takes place on a spherical substrate in the radial direction $(r)$. This process is expressed by Fick's equation in spherical coordinates

$\frac{\partial c_{i}}{\partial t}=D_{i}\left(\frac{\partial^{2} c_{i}}{\partial r^{2}}+\frac{2}{r} \frac{\partial c_{i}}{\partial r}\right)$

In this case, the solid growth front advances in the $r$-direction. Then, Eq. (11) is solved with the following boundary conditions:

$c_{i}(r, 0)=c_{i}^{0} ; \quad r \geq r_{0}$

$c_{i}[s(t), t]=0 ; \quad t>0$

$s(t)=r_{0}+\beta t$

$c_{i}(r, t)=c_{i}^{0} ; \quad r \rightarrow \infty$

$r_{0}$ being the initial radius of the substrate. The meaning of boundary conditions $((12 \mathrm{a})-(12 \mathrm{~d}))$ is the same as that indicated above for the plane plate substrate. The explicit solution of Eq. (11) 
with the conditions $((12 \mathrm{a})-(12 \mathrm{~d}))$ is

$c_{i}(r, t)=c_{i}^{0}-\frac{c_{i}^{0}}{r} \exp \left(-\frac{\beta}{2 D_{i}} \xi\right) \cdot\left[r_{0} \varphi(\xi, t)+\beta \int_{0}^{t} \varphi\left(\xi, t^{\prime}\right) \mathrm{d} t^{\prime}\right]$

where

$\xi=r-r_{0}-\beta t$

Plots of $c_{i}$ versus $\xi$ for $r_{0}=1 \mathrm{~cm}$ and different values of $\beta$ are shown in Fig. 3. The radial gradient of $c_{i}$ increases with $\beta$, the effect being more remarkable at longer $t$.

For the electrodeposition of reactant $i$ with the electric charge $z_{i}$ the instantaneous rate equation, expressed as the cathodic current density $j_{\mathrm{c}}(t)$, is

$j_{\mathrm{c}}(t)=z_{i} F D_{i}\left(\frac{\partial c_{i}}{\partial \xi}\right)_{\xi=0}=z_{i} F D_{i}\left(\frac{\partial c_{i}}{\partial r}\right)_{r=r_{0}+\beta t}$

Then, the differential expression of Eq. (13) for $\xi=0$ is

$$
\begin{aligned}
{\left[\frac{\partial c(r, t)}{\partial r}\right]_{\xi=0}=\frac{c_{i}^{0}}{r} } & \left\{1+\frac{\beta}{2 D_{i}}\left[r_{0}+\beta t\right]+r_{0} \frac{\beta}{2 D_{i}} \operatorname{erf}\left(\beta \sqrt{\frac{t}{4 D_{i}}}\right)+r_{0} \frac{2}{\sqrt{\pi D_{i} t}} \exp \left(-\frac{\beta^{2}}{4 D_{i}} t\right)\right\} \\
+ & \frac{c_{i}^{0}}{r}\left\{\int_{0}^{t} \frac{\beta}{2 D_{i}} \operatorname{erf}\left(\beta \sqrt{\frac{t^{\prime}}{4 D_{i}}}\right)+\frac{2}{\sqrt{\pi D_{i} t^{\prime}}} \exp \left(-\frac{\beta^{2}}{4 D_{i}} t^{\prime}\right) \mathrm{d} t^{\prime}\right\}
\end{aligned}
$$

and

$$
\begin{aligned}
\varphi(\xi, t)= & \frac{1}{2}\left\{\exp \left(-\frac{\beta}{2 D_{i}} \xi\right) \operatorname{erfc}\left(\frac{\xi-\beta t}{\sqrt{4 D_{i} t}}\right)\right. \\
& \left.+\exp \left(\frac{\beta}{2 D_{i}} \xi\right) \operatorname{erfc}\left(\frac{\xi+\beta t}{\sqrt{4 D_{i} t}}\right)\right\}
\end{aligned}
$$
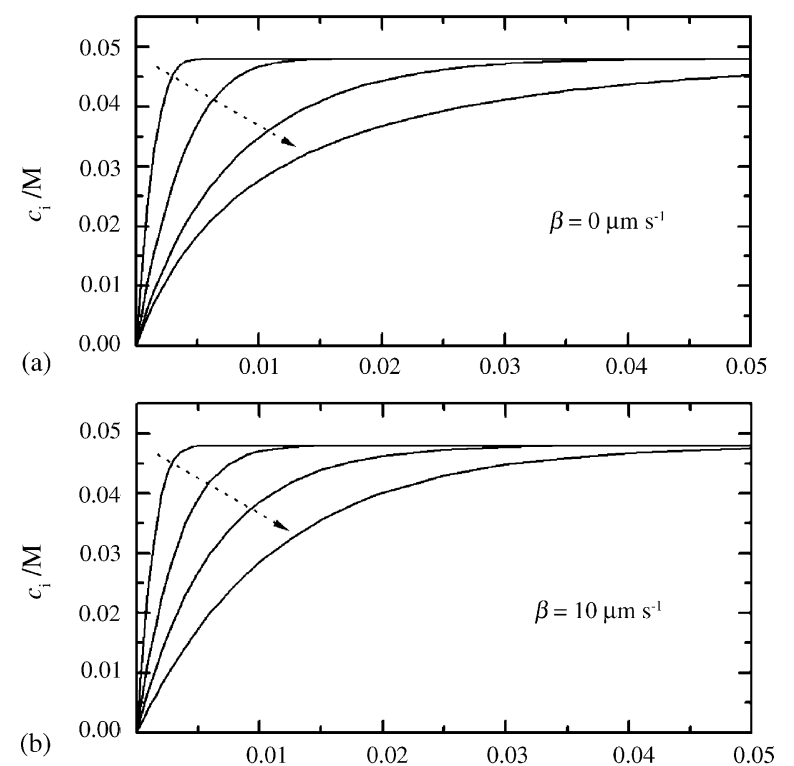$$
\text { (b) }
$$

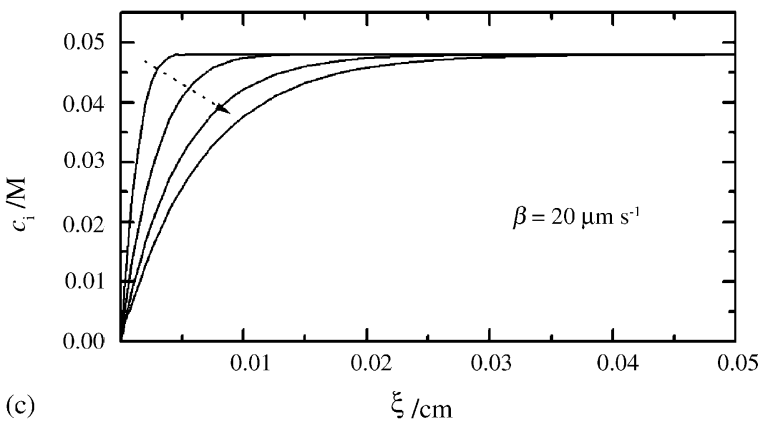

Fig. 3. (a-c) Plots of concentration profiles calculated for the sphere with Eq. (13) at different values of $\beta$ for $t=0.1,1,10$ and $100 \mathrm{~s}$, and $r_{0}=0.05 \mathrm{~cm}$. The value of $t$ increases as indicated by the arrows. The same values of $z_{i}, D_{i}$ and $c_{i}^{0}$ indicated in Fig. 1 were used. as the differential expression of Eq. (15) for $\xi=0$ is

$$
\left[\frac{\partial \varphi(\xi, t)}{\partial \xi}\right]_{\xi=0}=-\frac{\beta}{2 D_{i}} \operatorname{erf}\left(\frac{\beta t^{1 / 2}}{\sqrt{4 D_{i}}}\right)-\frac{1}{\sqrt{\pi D_{i} t}} \exp \left(-\frac{\beta^{2} t}{4 D_{i}}\right)
$$

To obtain the equation for $j_{\mathrm{c}}(t)$ it is convenient to define the following dimensionless variable:

$$
\tau=\frac{\beta^{2} t}{4 D_{i}}
$$

and

$$
\psi(\tau)=\operatorname{erf}(\sqrt{\tau})+\frac{1}{\sqrt{\pi \tau}} \exp (-\tau)
$$

By introducing first Eq. (17) in Eq. (16), and then considering Eqs. (19) and (20), the expression of $j_{\mathrm{c}}(t)$ results in

$$
\begin{aligned}
j_{\mathrm{c}}(\tau)= & \frac{1}{2} z_{i} F \beta c_{1}^{0}\left[1+\frac{r_{0}}{r} \psi(\tau)\right] \\
& +z_{i} F D_{i} \frac{c_{i}^{0}}{r}\left[1+\int_{0}^{\tau} \psi\left(\tau^{\prime}\right) \mathrm{d} \tau^{\prime}\right]
\end{aligned}
$$

According to Eq. (21) (Fig. 4a) the fastest decay of $j_{\mathrm{c}}(t)$ is observed initially, whereas for $t>700 \mathrm{~s}, j_{\mathrm{c}}(t)$ tends to attain a limiting value that increases with $r_{0}$. By setting $t$ at $1000 \mathrm{~s}$, a rapid increase in $\mathrm{d}\left[j_{\mathrm{c}}(t)\right] / \mathrm{d} r_{0}$ with $r_{0}$ is obtained. Likewise, the decay of $j_{\mathrm{c}}(t)$ exhibits a bump at about $100 \mathrm{~s}$ due to $\beta$. These bumps are more noticeable as $r_{0}$ is increased. Current density transients for $r_{0}=1 \mathrm{~cm}$ also depend on $\beta$ (Fig. 4b) and attain a limiting value that increases linearly with $\beta$, as occurs for the plane plate. In agreement with the advection contribution, the greater the value of $\beta$, the sooner the appearance of current density bumps (Fig. 4b, inset). For $r \rightarrow \infty$ the value of $j_{\mathrm{c}}(t)$ approaches Eq. (6) for the plane plate substrate.

On the other hand, the limit of Eq. (21) for $\tau \rightarrow \infty$ and $r \rightarrow \infty$ can be calculated considering the limit of Eq. (20) and the fact that the numerical integration of the second term of Eq. (21) for $\tau \rightarrow \infty$ approaches $\tau$. Then, the limit of Eq. (21) results in

$\lim \left[j_{\mathrm{c}}(\tau)\right]_{t \rightarrow \infty}=\frac{3}{4}\left(j_{\mathrm{c}}\right)_{\mathrm{adv}}$ 


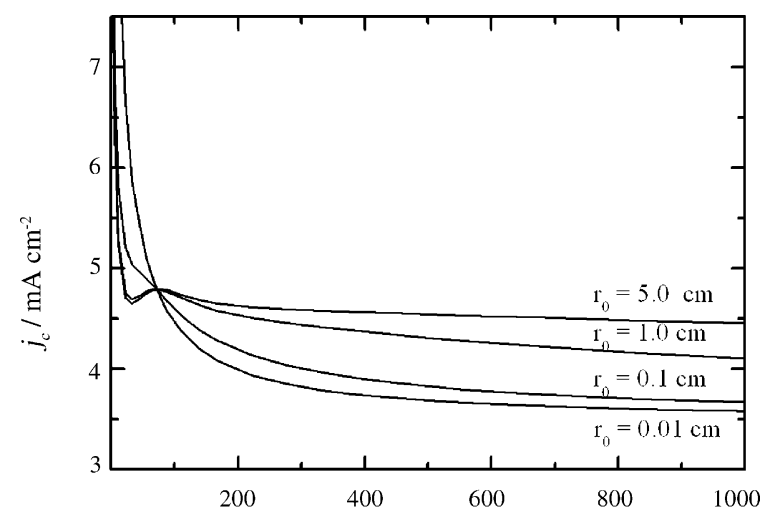

(a)

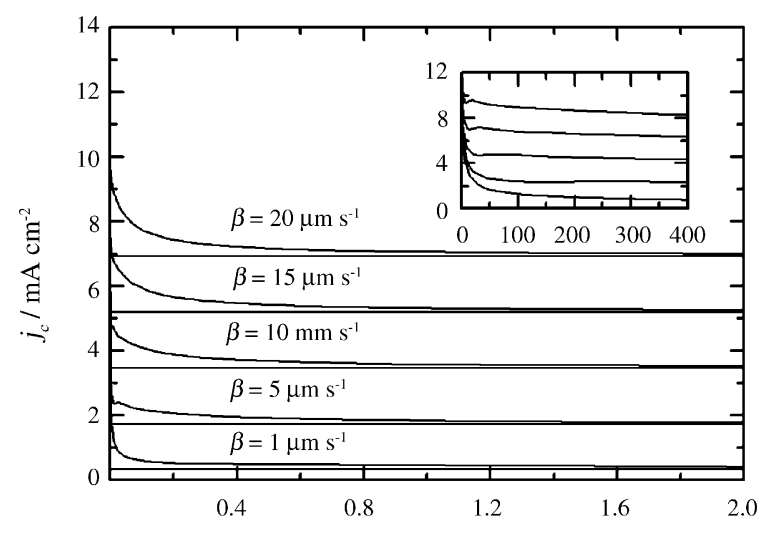

(b)

$10^{-4} t / s$

Fig. 4. (a) Plots of $j_{\mathrm{c}}$ vs. $t$ calculated for the sphere with Eq. (21) for $\beta=10 \mu \mathrm{m} \mathrm{s}^{-1}$ at different values of $r_{0}$, as indicated. Values of $z_{i}, D_{i}$, and $c_{i}^{0}$ are the same as those in Fig. 1. The current density bumps become more remarkable as $r_{0}$ is increased. (b) Plots of $j_{\mathrm{c}}$ vs. $t$ calculated with Eq. (21) for $r_{0}=1 \mathrm{~cm}$ at different values of $\beta$, as indicated. Values of $z_{i}, D_{i}$ and $c_{i}^{0}$ are the same as those in Fig. 1. The inset shows the same plots for a shorter range of time to distinguish the current density bumps.

\section{Experimental}

Potentiostatic current density transient data from silver dense branching pattern formation by electrodeposition from conventional aqueous plating solutions were utilised to test the corresponding theoretical equations derived for plane plate and spherical cathodes. The selected testing process offers a number of advantages for our purpose. It rapidly reaches a diffusioncontrolled kinetics due to the high exchange current density of the silver electrode in aqueous media [12-14]. The process starts with the nucleation and growth of silver islands a few monolayers in thickness that occur in a short time and involve a negligible charge as compared to that of the diffusion-controlled silver phase growth. Otherwise, the low, almost constant apparent density of silver dense branching patterns leads to a large almost constant growth front velocity closely approaching the Stefan condition. The almost constant roughness of the growth front and its small irregularities, most of them located within the thickness of the diffusion layer, makes it feasible to introduce a roughness correction factor in the evaluation of the cathodic current density. Finally, the use of a quasi-2D electrochemical cell for the plane plate cathode, and glycerol to increase the viscosity of the plating solution for the spherical cathodes, ensured a negligible contribution of free convection to the process, as required.

A quasi-2D-rectangular horizontal electrochemical cell was made by a parallel edge arrangement of two $99.9 \%$ purity silver sheets used as cathode and anode, respectively, with a cathodeto-anode separation distance $l_{\mathrm{c}-\mathrm{a}}=2 \mathrm{~cm}$. To avoid border effects and initially ensure a homogeneous primary current distribution on the cathode, a Teflon ${ }^{\circledR}$ mask $0.025 \mathrm{~cm}$ thick was symmetrically placed on both sides of the cathode. The top and the bottom of the cells consisted of two parallel either Lucite ${ }^{\circledR}$ or glass flat plates separated by the distance $l_{\mathrm{S}}=0.025 \mathrm{~cm}$.

The cylindrical electrochemical cell $1 \mathrm{~cm}$ in height consisted of a spherical cathode made by flame melting a $99.99 \%$ purity, $0.0125 \mathrm{~cm}$ radius platinum wire to produce a sphere with an initial radius $r_{0}=0.026 \mathrm{~cm}$. The cathode was placed at the centre of a silver ring anode suspended from the top of the cell. The anode was made of $99.9 \%$ purity silver sheet $\left(r_{\mathrm{a}}=1 \mathrm{~cm}\right)$. An optical quality plane glass at the bottom of the cell allowed following the evolution of the silver electrodeposit. Both cells were mounted on a suspended table to avoid the influence of spurious mechanical vibrations on the kinetics of the process.

The following plating solutions were utilised. Solution I: aqueous $0.024 \mathrm{M}$ silver sulphate $+0.5 \mathrm{M}$ sulphuric acid; solution II: aqueous $0.024 \mathrm{M}$ silver sulphate $+1 \mathrm{M}$ sulphuric acid; solution III: aqueous $0.024 \mathrm{M}$ silver sulphate $+1 \mathrm{M}$ sulphuric acid $+5 \mathrm{M}$ glycerol. The excess of sulphuric acid behaved as a supporting electrolyte to make the contribution of migration negligible. Glycerol-containing solutions behave as Newtonian fluids [15]. The viscosity $(\eta)$ correction to the diffusion coefficient of the reacting ion $\left(D_{i}\right)$ was made using the Walden ratio $\left(D_{i} \eta / T=5.53 \times 10^{-10} \mathrm{~cm}^{4} \mathrm{~s}^{-2} \mathrm{~K}^{-1}\right.$ at $\left.298 \mathrm{~K}\right)$. Solutions were prepared from a.r. chemicals and MilliQ ${ }^{\circledR}$ water. Runs were made at $25 \pm 1{ }^{\circ} \mathrm{C}$.

For each plating bath the cathodic polarization curves were run at $v=0.30 \mathrm{~V} / \mathrm{s}$ to determine the potential range of the mass transport kinetic regime. Polarization curves were plotted as cathode-to-anode voltage $\left(\Delta E_{\mathrm{c}-\mathrm{a}}\right)$ versus cathodic current $\left(I_{\mathrm{c}}\right)$. The effective cathodic potential was $\Delta E_{\mathrm{eff}}=\Delta E_{\mathrm{c}-\mathrm{a}}-I_{\mathrm{c}} R_{\mathrm{O}}, R_{\mathrm{O}}$ being the ohmic resistance between the cathode and the anode of the cell.

Silver patterns were grown at constant $\Delta E_{\mathrm{c}-\mathrm{a}}$ in the range $-0.90 \geq \Delta E_{\mathrm{a}-\mathrm{c}} \geq-0.20 \mathrm{~V}$. The pattern morphology and the silver growth front velocity were followed from photographs obtained with a stereoscopic microscope (Stemi 200 Zeiss) coupled to a Canon Powershot G 5 camera. Simultaneously, the cathodic current $\left(I_{\mathrm{c}}\right)$ and charge $\left(Q_{\mathrm{c}}\right)$ were recorded using a Radiometer 32 potentiostat.

\section{Results and discussion}

\subsection{Plane plate substrate}

The potentiostatic current transients (Fig. 5) exhibit a complicated behavior in the range $5-150$ s, i.e., in the interval where 


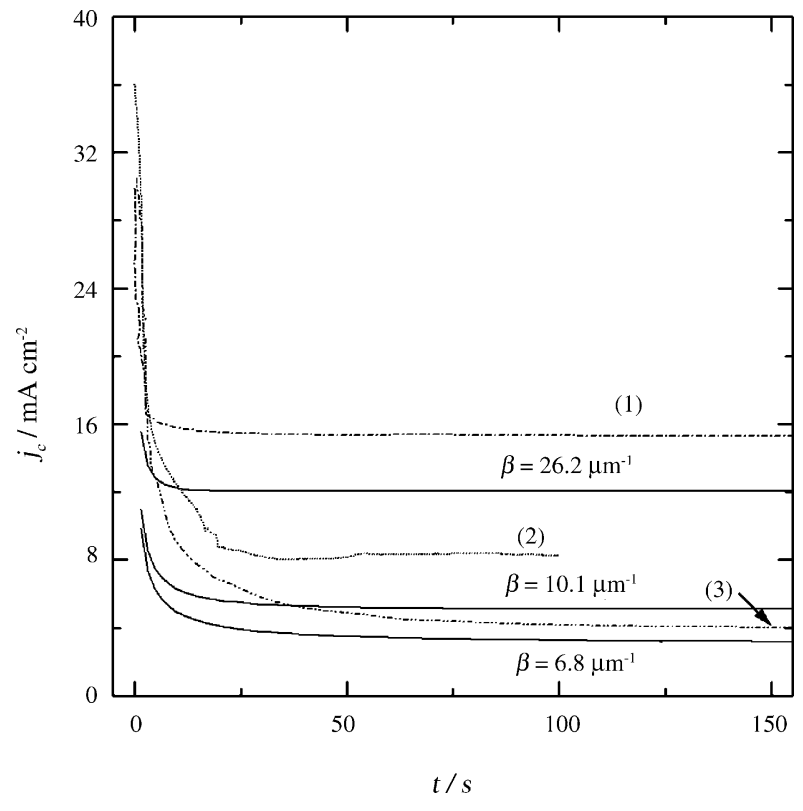

Fig. 5. Comparison of experimental (raw data) and theoretical plots of cathodic current density transients for silver electrodeposition from a planar plate cathode in aqueous $0.024 \mathrm{M}$ silver sulphate $+1 \mathrm{M}$ sulphuric acid. Experimental data: (1) $\Delta E_{\mathrm{c}-\mathrm{a}}=-0.700 \mathrm{~V}$ and $\beta=26.2 \mu \mathrm{m} \mathrm{s}^{-1}$; (2) $\Delta E_{\mathrm{c}-\mathrm{a}}=-0.600 \mathrm{~V}$ and $\beta=10.1 \mu \mathrm{m} \mathrm{s}^{-1}$; (3) $\Delta E_{\mathrm{c}-\mathrm{a}}=-0.475 \mathrm{~V}$ and $\beta=6.8 \mu \mathrm{m} \mathrm{s}^{-1}$. Theoretical data for $\beta=26.2,10.1$ and $6.8 \mu \mathrm{m} \mathrm{s}^{-1}$ (solid traces).

the influence of the Stefan effect becomes remarkable and then quasi-stationary advection sets in.

A sequence of photographs of quasi-2D silver electrodeposits grown on the plane plate cathode (Fig. 6a) shows the stochastic nature of silver electrodeposition at levels of the order of $10 \mu \mathrm{m}$. The apparent density and roughness of these electrodeposits for $t>60 \mathrm{~s} \mathrm{(Fig.} \mathrm{6b)} \mathrm{remain} \mathrm{almost} \mathrm{constant,} \mathrm{as} \mathrm{expected}$ for an isotropic growth. The roughness correction was estimated for a minimal and maximal situation, namely, when there is no interference of the surface regularities on the outer plane of the diffusion layer the roughness factor approaches one, whereas the maximal situation is given by the analysis of the electrodeposit cross-section through the value of the root mean square eight $[9,16,17]$. These roughness factors are comprised between 1.8 and 3.0, i.e., they are greater than those obtained from the ratio $j_{\mathrm{c}, \exp } / j_{\mathrm{c} \text {,the }}$ at time $t$. Therefore, to evaluate from pattern profiles how large the influence of the front roughness on the average diffusion layer thickness at the cathode is, let us compare the average protrusion height and cavity depth at the growth front, which are in the range $0.004-0.015 \mathrm{~cm}$, to the average diffusion layer thickness estimated from the ratio $\left\langle\delta_{\mathrm{N}}\right\rangle=D_{\mathrm{Ag}^{+}} / \beta$, where $D_{\mathrm{Ag}^{+}}=1.39 \times 10^{-5} \mathrm{~cm}^{2} \mathrm{~s}^{-1}$ is the silver ion diffusion coefficient in the aqueous solution, and $\beta$ is the average velocity of the growth front in the range $7 \leq \beta \leq 26 \mu \mathrm{m} \mathrm{s}^{-1}$. Accordingly, the value of $\left\langle\delta_{\mathrm{N}}\right\rangle$ is in the range $0.005-0.020 \mathrm{~cm}$. Then, the profile of the diffusion layer should be to some extent modulated by the irregularities of the front surface. This means that at the time scale of our measurements the stochastic formation of protrusion and cavities at the micro scale should actually produce a roughness,
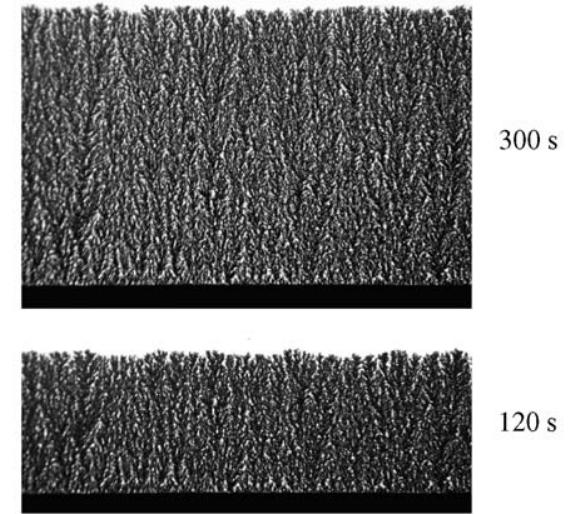

(a)
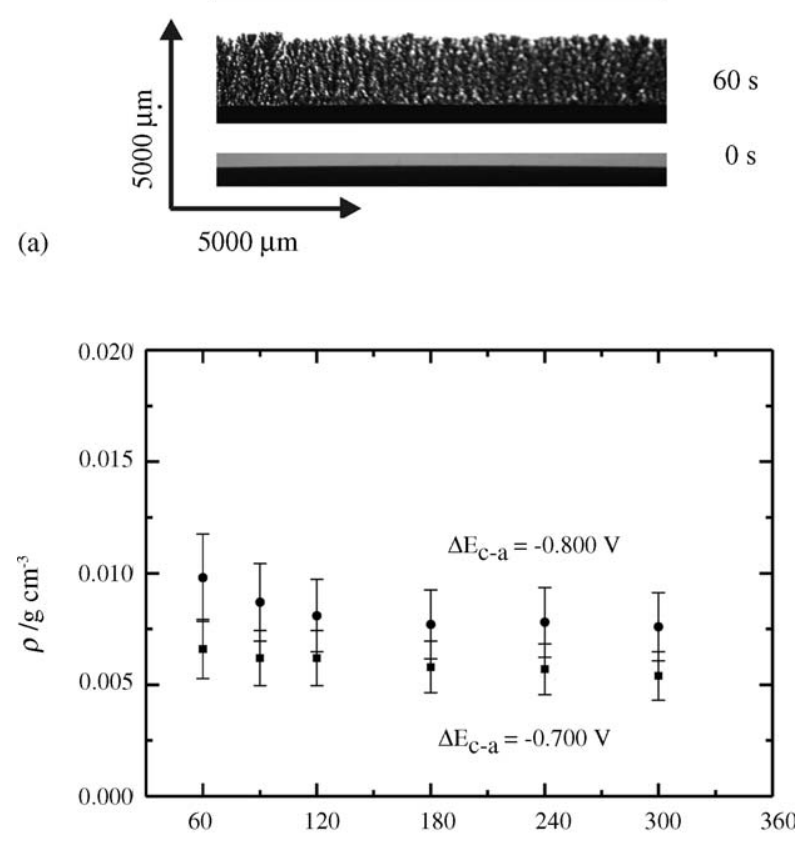

(b)

$t / s$

Fig. 6. (a) Sequence of silver growth patterns formed at the plane plate cathode in aqueous $0.024 \mathrm{M}$ silver sulphate $+1 \mathrm{M}$ sulphuric acid. $\Delta E_{\mathrm{c}-\mathrm{a}}=-0.700 \mathrm{~V}$. (b) Apparent density of silver electrodeposits vs. $t$ plots for $\Delta E_{\mathrm{c}-\mathrm{a}}=-0.700$ and -0.800 V. Error bars are indicated.

which is intermediate between that of the initial plane plate, and the true roughness of the growth front. Correspondingly, the average properties of the interface can be utilised to test the continuous, deterministic model of advection. At the scale of the quasi-stationary diffusion layer thickness the influence of the fractal surface of the electrodeposit becomes unnoticeable [18].

Experimental data, after the above correction, fulfill reasonably well the linear dependence $j_{\mathrm{c}}$ versus $\langle\beta\rangle$ plot predicted by the advection term in Eq. (7) (Fig. 7). This dependence contrasts the experimental $\langle\beta\rangle^{1 / 2}$ linear dependence that would result from laminar flow forced convection perpendicularly on a plane plate electrode $[19,20]$.

There is a good correlation of data resulting from different runs in terms of the dimensionless Eq. (10) (Fig. 8). The surprisingly good prediction for the dimensionless correlation indicates 


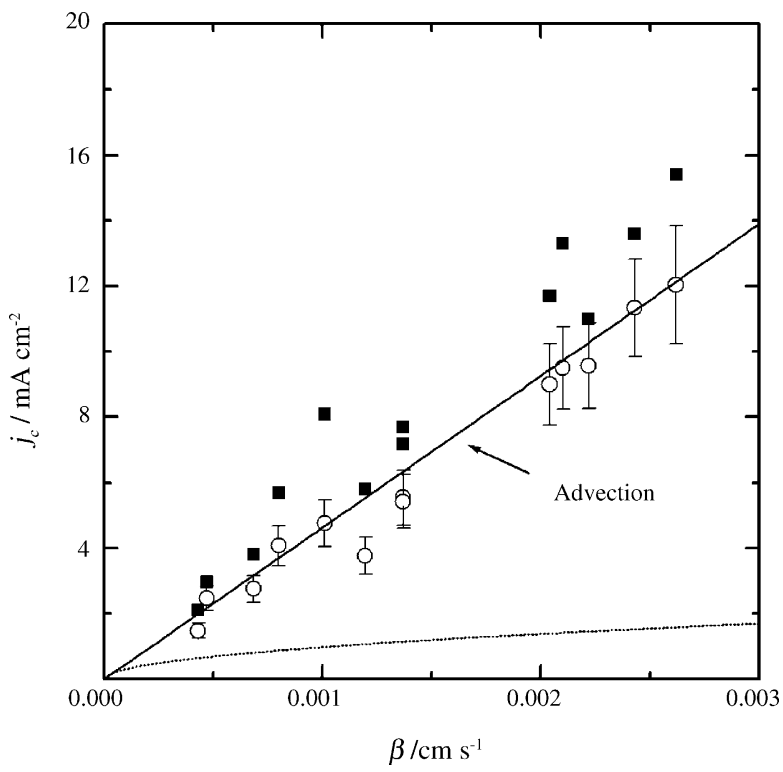

Fig. 7. Plot of $j_{\mathrm{c}}$ vs. $\beta$ : (四) raw data and $(\bigcirc)$ data after roughness correction. The straight line represents the advection term, and the lower trace corresponds to laminar flow forced convection.

that the influence of roughness on the $j_{\mathrm{c}}(\tau) /\left(j_{\mathrm{c}}\right)_{\mathrm{adv}}$ ratio tends to cancel.

\subsection{The spherical substrate}

The current transients (Fig. 9a) run on the spherical cathode involve the contribution of the increase in the surface area, which

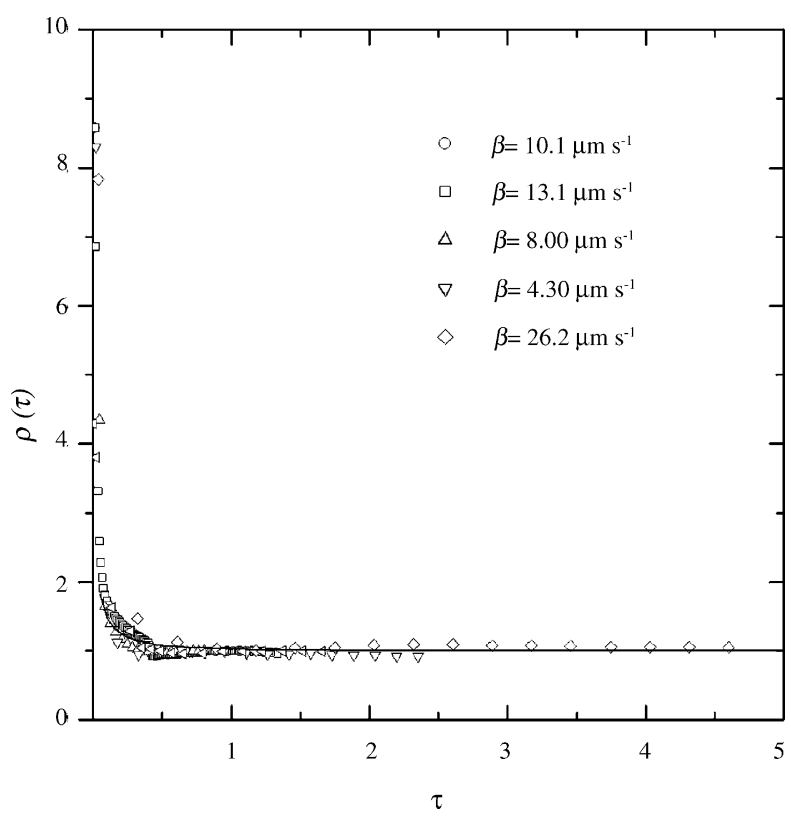

Fig. 8. Dimensionless plot of experimental potentiostatic cathodic current density transients for silver electrodeposition on the plane plate cathode. The full trace represents Eq. (10). Symbols correspond to different values of $\beta$, as indicated in the figure. (The permission of The American Chemical Society for reproducing this figure from reference 10 is acknowledged.)

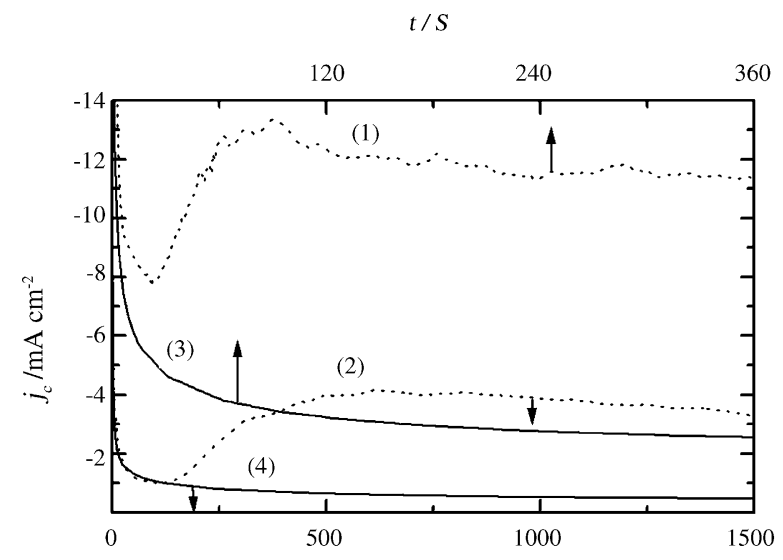

(a)

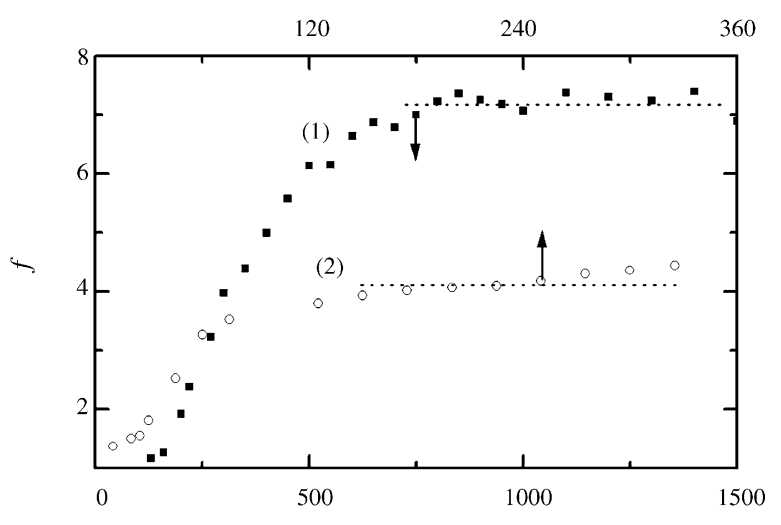

(b)

$t / S$

Fig. 9. (a) Potentiostatic current transient (raw data, dashed trace) for silver electrodeposition on a spherical cathode $\left(r_{0}=0.026 \mathrm{~cm}\right)$. Aqueous $0.024 \mathrm{M}$ silver sulphate $+1 \mathrm{M}$ sulphuric acid. (1) Glycerol-free and (2) $5 \mathrm{M}$ glycerol-containing solution. $\Delta E_{\mathrm{c}-\mathrm{a}}=-0.200 \mathrm{~V}$. The full traces (3) and (4) are calculated with Eq. (21), for the above-mentioned plating solutions, for $\beta=5$ and $1.3 \mu \mathrm{m} \mathrm{s}^{-1}$, respectively. (b) Plot of $f$ vs. $t$ for silver electrodeposits run at spherical cathodes with the plating solutions indicated in (a), in the absence of glycerol (1), and $5 \mathrm{M}$ glycerol-containing solution (2). The dashed traces indicate the interval in which a constant surface roughness electrodeposit is approached.

is accounted by the solution of Eq. (11), and a roughness modulation of the diffusion layer thickness similar to that described above for the plane plate cathode. However, there is an important difference between the plane plate and the spherical electrode as the application of the theory to experimental data becomes only possible for those electrodeposits approaching spherical shapes and constant roughness. In fact, in the absence of free convection silver dense branched electrodeposits tend to acquire a spherical symmetry for $t>100-400$ s for low viscosity plating solutions (Fig. 10a), and for $t>400-600 \mathrm{~s}$ for high viscosity plating solutions (Fig. 10b). For these values of $t$ the average growth pattern radius $(\langle r\rangle)$ increases linearly with $t$ (Fig. 11). Conversely, for $t>1500 \mathrm{~s}$, an apparent increase in roughness and a small decrease in the apparent density of growth patterns can be noticed making these data useless for our purpose. These changes can be due to a relevant contribution of free convection that turns the ball-shaped patterns into slightly conical ones 
(a)

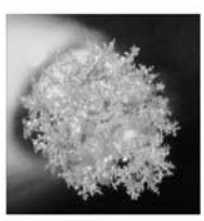

$90 \mathrm{~s}$

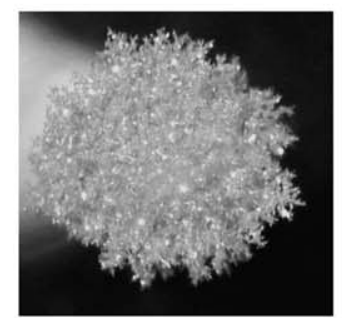

$150 \mathrm{~s}$

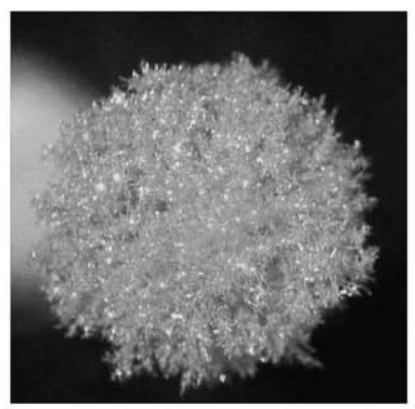

$240 \mathrm{~s}$

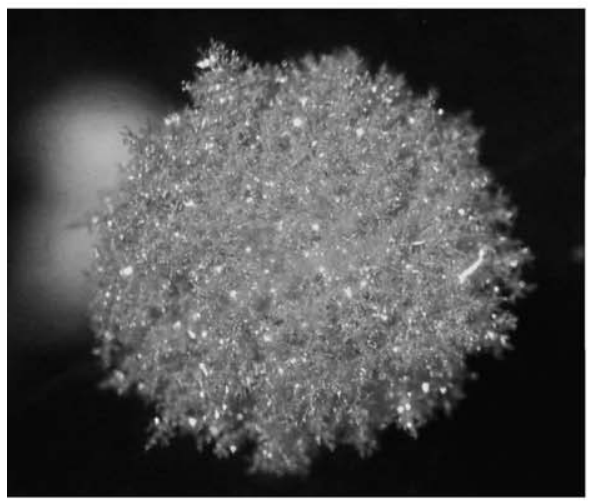

$330 \mathrm{~s}$ (b)

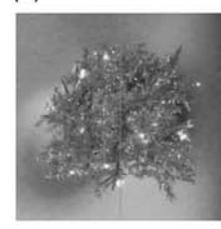

$300 \mathrm{~s}$

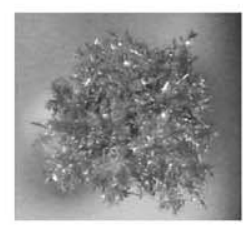

$360 \mathrm{~s}$

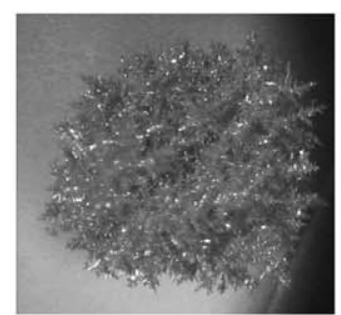

$600 \mathrm{~s}$

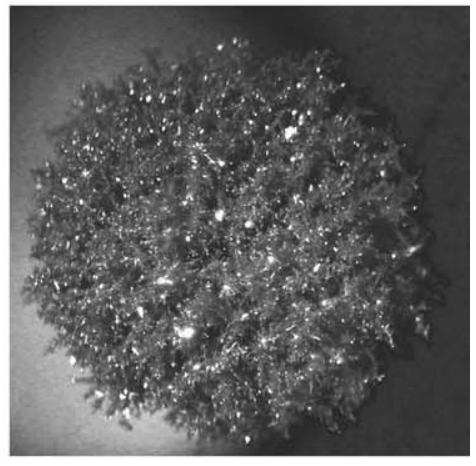

$1140 \mathrm{~s}$

$2500 \mu \mathrm{m}$

Fig. 10. Silver growth pattern sequence at the spherical $\left(r_{0}=0.026 \mathrm{~cm}\right)$ cathode in aqueous $0.024 \mathrm{M}$ silver sulphate $+1 \mathrm{M}$ sulphuric acid. $\Delta E_{\mathrm{c}-\mathrm{a}}=-0.200 \mathrm{~V}$. (a) Glycerol-free and (b) $5 \mathrm{M}$ glycerol.

$[20,21]$. The larger the initial radius of the cathode the higher the value of $j_{\mathrm{c}}$ and, correspondingly, the sooner the influence of free convection due to density gradients in the bulk of the solution.

The roughness factor $(f)$, calculated from the ratio $f_{2 \mathrm{D}}=j_{\mathrm{c}, \text { exp }} / j_{\mathrm{c}, \text { the }}$, begins to increase after exceeding an induction time $t_{\text {in }} \cong 40$ s for low viscosity solutions and $t_{\text {in }} \cong 200 \mathrm{~s}$ for high viscosity solutions (Fig. 9b). These values of $t_{\text {in }}$ could be assigned to the time required to achieve a cathode completely silvered [22]. Later, the $f$ versus $t$ plots attain an almost constant value within a certain range of $t$ that agrees with that in the $\langle r\rangle$ versus $t$ plots where macroscopic growth patterns approach the spherical shape (Fig. 11). The roughness factor correction, as applied to the plane plate cathode can be extended to the spherical cathode only for the range of $t$ in which a sphere-shaped electrodeposit is approached and its radius increases linearly with $t$. Under these circumstances, the apparent density of the electrodeposit becomes constant and the average roughness $\langle f\rangle$ in this range of $t$ (Fig. 9b) can be taken as the correction factor for $j_{c, \text { exp }}$. Accordingly, a fairly good agreement between the theoretical and experimental current density transients, irrespective of the viscosity of the plating solution can be obtained (Fig. 12). 


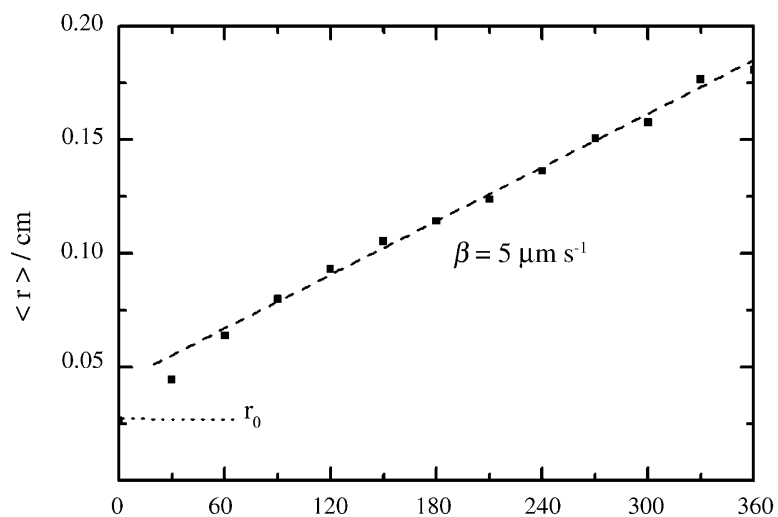

(a)

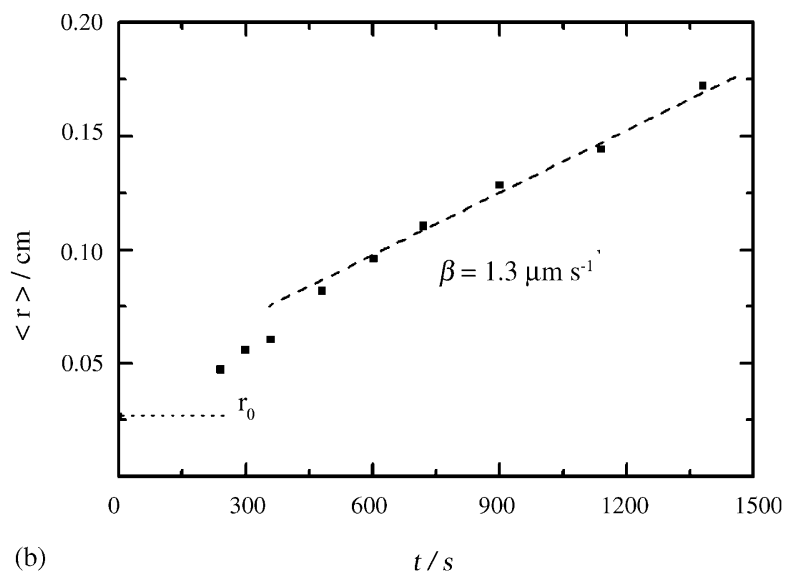

Fig. 11. Plot of $\langle r\rangle$ vs. $t$ from silver growth patterns produced at the spherical cathode of $r_{0}=0.026 \mathrm{~cm}$. Aqueous $0.024 \mathrm{M}$ silver sulphate $+1 \mathrm{M}$ sulphuric acid. $\Delta E_{\mathrm{c}-\mathrm{a}}=-0.200 \mathrm{~V}$. (a) Glycerol-free and (b) $5 \mathrm{M}$ glycerol. The linear portion of the $r$ vs. $t$ plots are indicated by the dashed traces. The horizontal trace corresponds to the value of $r_{0}$.

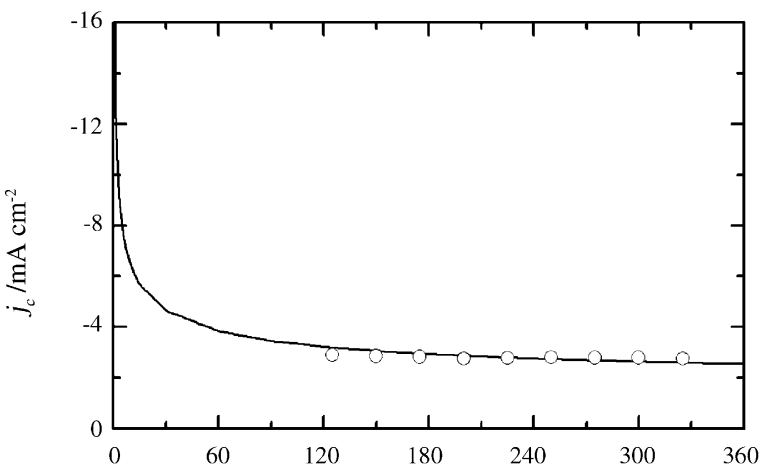

(a)

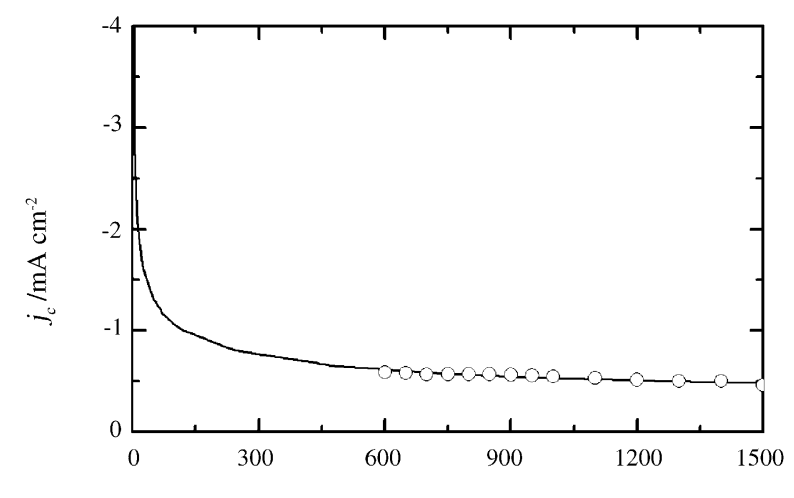

(b)

Fig. 12. Correlation of current density transient experimental data in the advection regime from the spherical cathode, after roughness correction, and the predictions of Eq. (21). Silver electrodeposition from aqueous $0.024 \mathrm{M}$ silver sulphate $+1 \mathrm{M}$ sulphuric acid. $\Delta E_{\mathrm{c}-\mathrm{a}}=-0.200 \mathrm{~V}$. (a) glycerol-free $\left(\beta=5 \mu \mathrm{m} \mathrm{s}{ }^{-1}\right)$ and (b) $5 \mathrm{M}$ glycerol $\left(\beta=1.3 \mu \mathrm{m} \mathrm{s}^{-1}\right)$.

\section{Conclusions}

The solutions of the Stefan problem, as presented, are of special interest to understand mass transport mechanisms at moving boundary interfaces. The rate equations resulting for a plane plate and a spherical geometry converge for the limiting conditions derived for $r \rightarrow \infty$. For both substrate geometries, the solid growth under mass transfer kinetics and linear displacement of the interfacial boundary with time, in the absence of convection and surface roughness effects, involves the simultaneous contribution of diffusion and advection. The contribution of advection to the kinetics becomes more remarkable when the duration of the process is longer and the radius of the surface increases. For both geometries the solid growth process exhibits a transition in kinetic control from diffusion to advection.

Results reported in this work indicate the possible significance of advection in a variety of phenomena covering a wide range of space and temporal scales, including deviations from classical kinetic relationships, such as Sand equation [9,23], when moving boundaries are involved.

\section{Acknowledgments}

This work was financially supported by the Consejo Nacional de Investigaciones Científicas y Técnicas (CONICET), the Universidad Nacional de La Plata, Argentina, and the Pontificia Universidad Católica Argentina, Buenos Aires, Argentina.

\section{References}

[1] V.E. Levich, Physicochemical Hydrodynamics, Englewood Cliffs, New Jersey, 1962 (Chapter 2).

[2] D. Tarzia, Seminario sobre el Problema de Stefan y sus Aplicaciones, Institute of Mathematics "Bepo Levi", Rosario, Argentina, 1984.

[3] D.G. Wilson, A.D. Solomon, P.T. Boggs (Eds.), Moving Boundaries Problems, Academic Press, New York, 1978.

[4] J. Stefan, Zber. Akad. Wiss. Wien. 98 (1889) 473.

[5] J. Stefan, Zber. Akad. Wiss. Wien. 98 (1889) 965.

[6] J. Stefan, Ann. Phys. Chem. 98 (1889) 473.

[7] J. Crank, Free and Moving Boundary Problems, Clarendon Press, Oxford, 1984.

[8] D.P. Barkey, in: R.C. Alkire, D.M. Kolb (Eds.), Advances in Electrochemical Science and Engineering, J. Wiley/VCH, New York/Frankfurt a/M, 2002, p. 154. 
[9] M.P. Pasquale, S.L. Marchiano, A.J. Arvia, J. Electroanal. Chem. 532 (2002) 255.

[10] M.P. Pasquale, S.L. Marchiano, J.L. Vicente, A.J. Arvia, J. Phys. Chem. B 108 (2004) 13315.

[11] J.S. Newman, Electrochemical Systems, second ed., Prentice-Hall, Englewood Cliffs, NY, 1991.

[12] A.J. Bard (Ed.), The Encyclopedia of the Electrochemistry of the Elements, vol. 8, Marcel Dekker, New York, 1973.

[13] W. Budevski, E. Bostanov, T. Vitanov, Z. Stoinov, A. Kotzewa, R. Kasichev, Electrochim. Acta 11 (1966) 1697.

[14] J.D. Porter, T.O. Robison, J. Phys. Chem. 97 (1993) 6696.

[15] A.J. Arvia, J.C. Bazán, J.S.W. Carrozza, Electrochim. Acta 11 (1966) 881.

[16] A.L. Barabasi, H.E. Stanley, Fractal Concepts in Surface Growth, Cambridge University Press, Cambridge, 1995.
[17] P. Schilardi, S.L. Marchiano, R.C. Salvarezza, A. Hernández Creus, A.J. Arvia, J. Electroanal. Chem. 431 (1997) 81.

[18] J.S. Andrade, M. Filoche, B. Sapoval, Europhys. Lett. 55 (2001) 573.

[19] S.L. Marchiano, A.J. Arvia, Electrochim. Acta 12 (1967) 801.

[20] J.C. Bazán, S.L. Marchiano, A.J. Arvia, Electrochim. Acta 12 (1967) 821.

[21] A. Hernández Creus, P. Carro, S. González, A.E. Bolzan, R.C. Salvarezza, S.L. Marchiano, A.J. Arvia, J. Electroanal. Chem. 336 (1992) 85.

[22] P. Carro, S. Ambrosolio, S.L. Marchiano, A. Hernández Creus, R.C. Salvarezza, A.J. Arvia, J. Electroanal. Chem. 396 (1995) 183.

[23] H.J.S. Sand, Philos. Mag. 1 (1901) 45. 